\title{
Transmission Tower Vibration Analysis System Based on Internet of Things
}

\author{
Wang Zhangqi, Wang Jian, and Zuo Li
}

\begin{abstract}
A vibration analysis system for transmission tower based on the Internet of Things is described in this paper. According to layering architecture concept of the Internet of Things, the system consists of three parts, which can be divided into sensing network, data communication and system software. The sensing network includes vibration acceleration transducer and some other measuring sensors, WiFi technology is used for data communication in the sensing network. The software module is responsible for storage, analysis and processing of the detected data. The system software mainly has the functions of the real-time vibration data collection, data filtering and time-frequency domain analysis. $C / S$ and $B / S$ architectures are constructed for remote display of vibration data. Finally, this system is utilized to perform vibration analysis for a guyed tower and a steel tube tower, and the method to assess the health level of the tested model is discussed.
\end{abstract}

Index Terms - The internet of things, transmission tower, vibration monitoring.

\section{INTRODUCTION}

The Internet of Things (or IoT for short) is a new generation of technology, which expands the Internet and constructs an information exchanging network among things. IoT features the ability of sensing, reliable transmission and intelligent process [1]. The information integrity will be greatly enhanced by applying IoT into the Smart Grid [2]. IoT can acquire, transmit and process the information etc. Some associated specialists have done certain expansion research based on the characteristics of the new technology and apply it to the monitoring of the transmission lines [3]. It is practical and progressive to apply the technology of IoT into the system of monitoring and analysis of transmission towers.

Transmission towers are the core of the power grid, it is necessary to keep the towers which support the conductors off the ground working safely and stably. When failure occurs to some components of tower, such as bolt loosens, angle iron failure etc., the stability and the longevity of the structure will decrease. In real situations, the failures may be difficult to recognize through manual check because the tower is big and the number of components is numerous. It is impractical to check all the bolts and joints in the tower. Recently, some useful methods of checking the healthy level of the tower are studied. The whole or partial vibration features maybe a good reflection of the structure system,

Manuscript received July 15, 2015; revised October 16, 2015.

The authors are with the Department of Mechanical Engineering, North China Electric Power University, Baoding 071003 Hebei Province, China (e-mail: wangzq2093@163.com, ncepu_wj@163.com, 317546383@qq.com). which means whether the tower is in good condition or not can be checked through monitoring and analyzing the vibration, which can be collected via accelerometers. T. Yin etc. introduce a dynamic reduction-based structural damage detection method for transmission towers utilizing ambient vibration data [4], [5]. Scott W. Doebling etc. give some advices of vibration-based damage identification methods [6].

The Internet of things is a new technology which simplifies and normalizes the architecture of monitoring and analysis system. Some technologies brought within the IoT make the construction of the transmission tower vibration analysis system more modern. To construct the new generation smart grid, combining the IoT technology with the transmission tower vibration analysis system is a promising trend. The smart grid demands the transmission towers' failure malfunction probability comes down to a minimum level.

This paper focuses on the application of monitoring and analysis of transmission towers using IoT technology and the construction of an experimental vibration analysis system. Firstly some concepts of the Internet of Things are introduced, and then the layering architecture of transmission tower monitoring system is proposed and established successfully. To check the feasibility of the system, some experiments on a guyed tower and a steel tube tower are done and the results show that the monitoring system can gain the dynamic characteristic far away from the site effectively.

\section{THE LAYING ARCHITECTURE OF THE ANALYSIS SYSTEM FOR TRANSMISSION TOWER VIBRATION BASED ON THE INTERNET OF THINGS}

The Internet of Things (IoT) which was proposed by Kevin Ashton refers to uniquely identifiable objects and their virtual representations in an Internet-like structure. In short, it is a kind of network which can identify the things. IoT is smarter and it provides users with more convenient access to the things in the network and this realizes the function of remote monitoring of transmission towers.

The architecture of layering is helpful to display IoT more clearly. IoT can be divided into three parts as sensing layer, network layer and application layer [7]. The vibration analysis system for transmission tower is designed based on the layering architecture concept of the Internet of things, which includes three parts, i.e. sensing network, communication technology and software application. And the architecture is shown in Fig. 1.

The sensing layer is mainly in charge of measuring physical signals and doing some simple processing. After 
some data processing, the raw data will be transformed into binary codes which can be transmitted easily. The data transmission network is extended by the terminal equipment in the network layer [8]. The terminal equipment is decided by the need of communication distance and data transfer rate. Different terminal device is constructed upon different communication protocol.

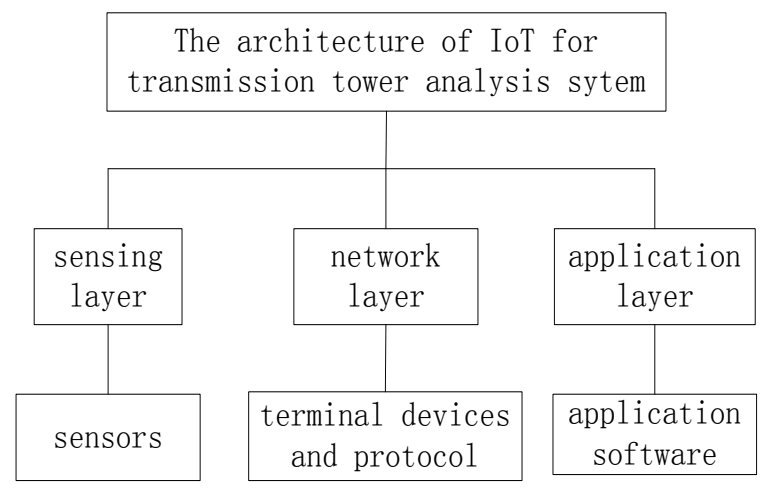

Fig.1. The architecture of IoT for transmission tower analysis system.

In the network layer, data is transferred from the sensing layer to the application layer [9]. Network layer, acting as a bridge link, is the place where data is transferred from sending layer to application layer. The network is composed by cable network, wireless network, network management system and cloud computing. The corresponding network communication protocol can ensure the data transmission. A transmission tower which is several kilometers far away from the monitoring center needs optical fiber communication when the data transfer rate is very high, while if the rate is low, GPRS mode can be chosen. Some close communication demand may use Zigbee, WiFi etc. instead. WiFi has a higher communication rate and Zigbee has the advantages of low power consumption. All the communication methods in the network layer can be summarized as communication terminal device and protocol.

The application software is on the application layer, which is the interface between users and system. It can serve for the concrete application and intelligentize it in some extent [10]. The layer can enhance the experience of the users, which realizes the interaction between individuals and IoT. There are many factors should be considered in this layer and the most important one is that users can implement what they need more conveniently.

The most important parts of the analysis system for the transmission tower include three modules, which are sensor network, communication technology and software application.

\section{A. Sensing Network}

The main object of sensing network is to gather the required data. To achieve this goal, the hardware of this network is equipped with transducers and wired or wireless communication facilities. Data collection equipment uses transducers to gain the required data. And the schematic diagram of data acquiring equipment is shown in Fig. 2.

In this transmission tower vibration monitoring system, some sensors are chosen for gathering on-site operation data. The details of the sensors, including types, functions and parameters are depicted in Table I.

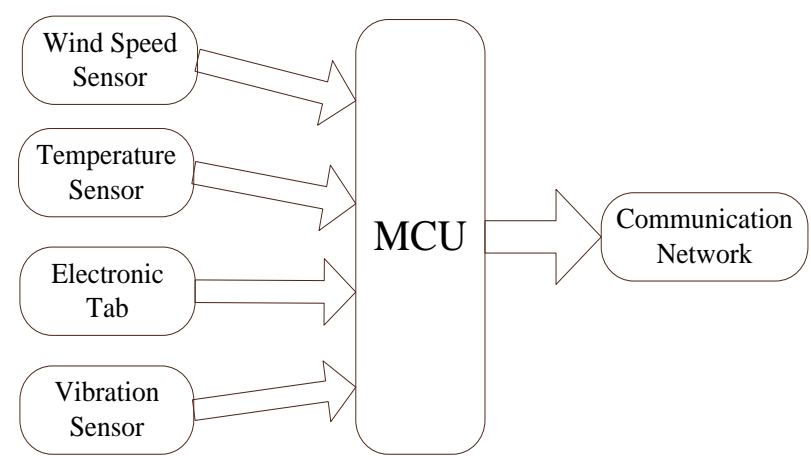

Fig. 2. Schematic diagram of data acquiring equipment.

TABLE I: SENSOR TYPES, FUNCTIONS AND PARAMETERS

\begin{tabular}{|c|c|c|}
\hline Sensor type & $\begin{array}{l}\text { Sensor } \\
\text { function }\end{array}$ & Main features of the sensors \\
\hline $\begin{array}{l}\text { Anemorumbo- } \\
\text { meter }\end{array}$ & $\begin{array}{l}\text { Measuring } \\
\text { wind speed } \\
\text { and direction }\end{array}$ & $\begin{array}{l}\text { Power supply:220VAC/50Hz or } \\
\text { 12VDC; } \\
\text { Wind speed measurement range: } 0 \sim \\
50 \mathrm{~m} / \mathrm{s}, 0 \sim 12 \text { levels; } \\
\text { Wind velocity measurement } \\
\text { precession: } \pm \text { (0.5+0.05 } \\
\text { V)m/s(V-real wind velocity); } \\
\text { Wind orientation measurement } \\
\text { range:0 } 360^{\circ} ; \\
\text { Wind orientation measurement } \\
\text { precision: } \pm 11.25^{\circ}\end{array}$ \\
\hline $\begin{array}{l}\text { AD590 } \\
\text { temperature } \\
\text { transducer }\end{array}$ & $\begin{array}{l}\text { Measure the } \\
\text { temperature }\end{array}$ & $\begin{array}{l}\text { Power supply:3 30V; } \\
\text { Output } \\
\text { current: } 223 \mu \mathrm{A}\left(-50{ }^{\circ} \mathrm{C}\right) \sim 423 \mu \mathrm{A}(+150 \\
\left.{ }^{\circ} \mathrm{C}\right) ; \\
\text { Sensitivity: } 1 \mu \mathrm{A} /{ }^{\circ} \mathrm{C}\end{array}$ \\
\hline $\begin{array}{l}\text { HS1101 } \\
\text { capacitive } \\
\text { humidity } \\
\text { sensor }\end{array}$ & $\begin{array}{l}\text { Measure the } \\
\text { humidity }\end{array}$ & $\begin{array}{l}\text { Power supply:5VDC; } \\
\text { Type output: } \sim 20 \% \mathrm{RH} \text { as } 1 \sim \\
\text { 1.6VDC }\end{array}$ \\
\hline $\begin{array}{l}\text { DH5906 } \\
\text { wireless } \\
\text { vibration } \\
\text { acquisition } \\
\text { module }\end{array}$ & $\begin{array}{l}\text { Measure the } \\
\text { vibration }\end{array}$ & $\begin{array}{l}\text { Sample frequency: } 2 \mathrm{~Hz} 、 5 \mathrm{~Hz}, 10 \mathrm{~Hz} \text {, } \\
20 \mathrm{~Hz}, 50 \mathrm{~Hz}, 100 \mathrm{~Hz}, 200 \mathrm{~Hz} \text {; } \\
\text { A/D resolution: } 16 \text { bit A/D convertor; } \\
\text { Systematic uncertainty: less than } 3 \% \\
\text { (FULL); } \\
\text { Wireless communication distance: } \\
\text { reliable transmission distance is } \\
\text { greater than } 200 \mathrm{~m} \text { in the case of } \\
\text { accessibility }\end{array}$ \\
\hline $\begin{array}{l}\text { RFID } \\
\text { anti-metal tag }\end{array}$ & $\begin{array}{l}\text { Identify the } \\
\text { tower } \\
\text { information }\end{array}$ & $\begin{array}{l}\text { Frequency:860-960MHz; } \\
\text { Memory:96 Bits or extended to } 512 \\
\text { Bits }\end{array}$ \\
\hline
\end{tabular}

\section{B. Communication Technology}

The monitoring data of transmission towers need to be transmitted to the monitoring station through communication technology, then the monitoring station conduct the condition assessment with the predefined parameters. In this system we chose $\mathrm{WiFi}$ wireless communication protocol to transmit data because the vibration data rate is high.

\section{Software Application}

The software application is constructed by on-site segment, control segment and application segment, corresponding to the sensing layer, network layer and application layer of the Internet of Things respectively. The on-site segment is used for collecting the data. The control segment implements the data transmission from tower to monitoring center. The application software analyzes the data and calls the expert system procedure to estimate whether the damage occurs.

The architecture diagram of the vibration monitor system 
based on IoT is shown in Fig. 3.

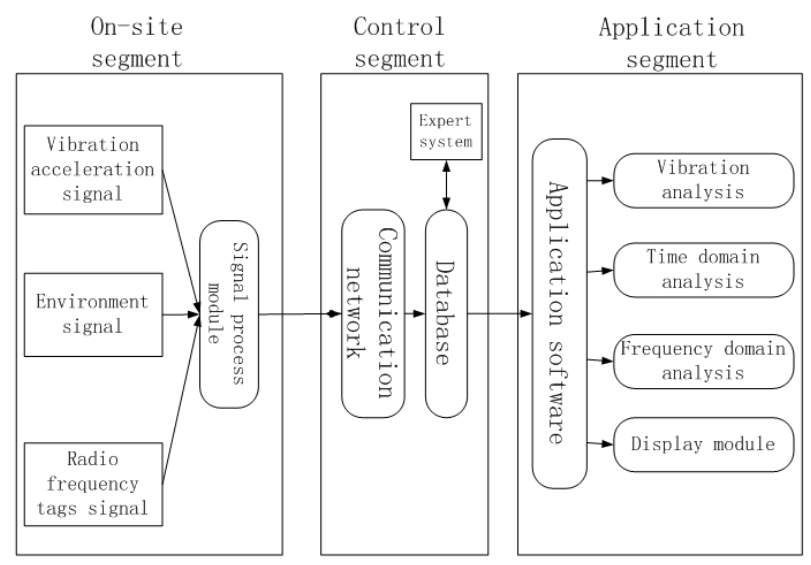

Fig. 3. Architecture diagram of the vibration monitor system based on IoT.

Database shown in Fig. 3 is constructed by SQL Server 2005 which provides a full-scale database operation. The software of data acquisition and analysis is programmed by LabVIEW (Laboratory Virtual Instrument Engineering Workbench). On this programming platform, some practical application can be edited simply.

\section{TRANSMISSION TOWER VIBRATION MONITORING AND ANALYSIS SYSTEM}

Transmission tower vibration monitoring and analysis system is constructed based on the concept of layering. It mainly consists of three modules, including data acquiring module, data process and analysis module, remote display module.

\section{A. Data Acquiring Module}

Data acquiring module is compiled by graphical program language LabVIEW. The data acquiring mode of one channel \& $\mathrm{N}$ sampling is applied in this data acquiring module. The acquired data is stored by storage program and named by the collection time.

The acquired data is stored by storage program and named by the collection time. All of the data is stored in the hard disk of the computer with the form of .lvm.

\section{B. Data Process and Analysis Module}

\section{1) Filter process}

The purpose of filter is to remove noise from the signal. The collected signal may contain a series of noise from electronic device, hence filter is needed in order to gain available signal. In this system, low-pass filter is adopted to gain the wanted transmission tower vibration signals.

\section{2) Time frequency domain analysis}

After time-frequency domain analysis of the acquired data, the vibration characteristics of the tower can be calculated. Frequency transformation is one of the most popular methods of fault diagnosis. When some faults occur, the frequency form of the signal may change simultaneously. So some latent defects can be detected though the analysis of the vibration frequency. In this system, the classical fast Fourier transformation (FFT) is used to gain the frequency domain features of the towers.

FFT is an algorithm to compute the discrete Fourier transform and its inverse. The main function of FFT is that it can calculate one time domain signal into a frequency one. In the time domain, transmission tower vibration is very complex and the signals usually perform a series of irregular curves. While the vibration feature in the frequency domain may reveals some potential faults in the structure.

\section{Remote Display}

The remote display function affords the operators with the interface of monitoring the on-site condition remotely, which plays an important role in the whole system. All the useful information for the tower operation status was displayed for the monitors directly. This function makes the judgments of the on-site status more reasonable. The remote connection is designed to construct the data connection between the main monitor and the on-site device. $\mathrm{B} / \mathrm{S}$ and $\mathrm{C} / \mathrm{S}$ display modes are realized here to enlarge the compatibility of the system.

$\mathrm{C} / \mathrm{S}$ mode, known as client/server mode, is a computer network mode between client and server, which assigns the application tasks to the client and server respectively. C/S mode can be realized by Datasocket technology provided by LabVIEW. The schematic diagram is shown in Fig.4(a).

$\mathrm{B} / \mathrm{S}$ mode is a browser/server mode with a core technology of web. TCP/IP and HTTP protocol is adopted to simplify the access to the monitor system. Users can visit the website on a frequently-used browser with permission. The schematic diagram is shown in Fig. 4(b).

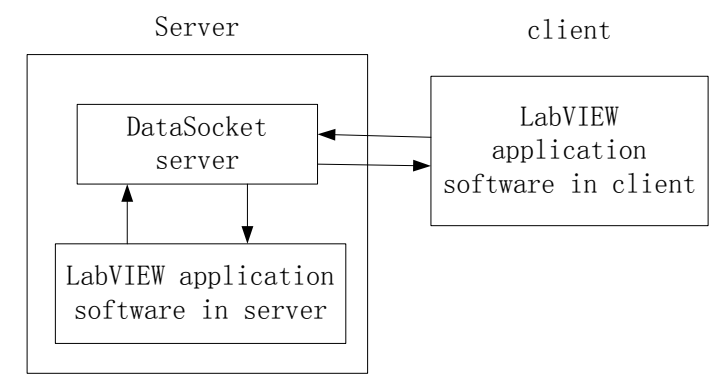

(a) Schematic diagram of $\mathrm{C} / \mathrm{S}$ mode

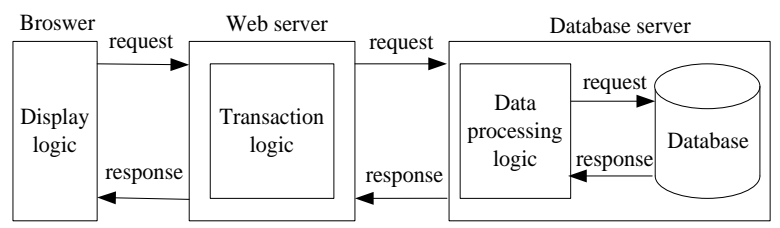

(b) Schematic diagram of $\mathrm{B} / \mathrm{S}$ mode

Fig. 4. Schematic diagram of two modes.

\section{TRANSMISSION LINES VIBRATION MONITORING AND ANALYSIS EXPERIMENTS}

To verify the monitoring system, two towers are experimented which are a steel tube tower and a guyed tower. Firstly, the factors which influence the vibrational characteristics of the tested tower model with methods of system dynamics are proposed. And then the towers are tested in real environment. The natural frequency of the towers can be achieved after data processing which is done by the system software. Combined with the theoretical derivation, the method of healthy diagnose is given.

\section{A. Transmission Tower Vibration Model}

The forced vibration equation could be expressed in the 
following equation:

$$
[M]\{\ddot{y}\}+[C]\{\dot{y}\}+[K]\{y\}=\{Q(t)\}
$$

Where $[M],[C]$ and $[K]$ are the mass matrices, damping matrices and stiffness matrices, respectively, $\{\ddot{y}\},\{\dot{y}\}$ and $\{y\}$ are acceleration vector, velocity vector and displacement vector; and $\{Q(t)\}$ is the exciting force vector. The corresponding free vibration equation of non-damping system is expressed as follows:

$$
[M]\{\ddot{y}\}+[K]\{y\}=\{0\}
$$

The characteristic equation of the Eq. (2) is shown in Eq. (3)

$$
\Delta\left(\omega_{\mathrm{n}}^{2}\right)=\operatorname{det}\left([K]-\omega_{\mathrm{n}}^{2}[M]\right)=0
$$

And the former $\mathrm{n}$ order natural frequencies, expressed as $\omega_{1} 、 \omega_{2} \ldots \omega_{\mathrm{n}}$, can be obtained from the Eq. (3). For the general engineering problems, the damping matrices could be processed into the formation of proportional damping as follows:

$$
[C]=a[M]+b[K]
$$

where $a$ and $b$ are the constant of proportionality. Substituting Eq. (4) into Eq. (1) and then using the orthogonality of the main modes, the damping natural frequency of the system can be calculated as follows:

$$
\omega_{\mathrm{d}_{i}}=\omega_{i} \sqrt{1-\varsigma_{i}^{2}}(i=1,2 \ldots n)
$$

where $\varsigma_{i}=\frac{a+b \omega_{i}^{2}}{2 \omega_{i}}$

Hence, the natural frequencies of the system are determined by the system mass distribution, system structure and elements connection, which can be described by mass matrices, damping matrices and stiffness matrices.

\section{B. Transmission Tower Vibration Monitoring and Analysis}

The transmission tower analysis system developed is then put into use to monitor two towers operation status. The tower vibration data is collected by wireless vibration transducer and then send to DWL-2100AP. FFT transfers the time domain signals into the frequency domain ones and the characteristics in the frequency domain show some intrinsic features of the transmission towers.

The spot map of the guyed tower is shown in Fig. 5(a). The vibration collection modules are installed at the height of $1.0 \mathrm{~m}$ and $4.5 \mathrm{~m}$ from the ground. The free vibration data are shown in Fig. 5(b), and the upper half is the vibration data at the height of $1.0 \mathrm{~m}$ while the lower one is $4.5 \mathrm{~m}$.

Frequency domain analysis is done with the collected vibration signal of the height of $1.0 \mathrm{~m}$ and the vibration feature of the guyed tower is shown in Fig. 6.

The experiment is done for the vertical flexible steel tube tower too. The radius of the steel tube tower is smaller with the height of the tower. Two wireless vibration collection modules are placed on the tower on the height of $1.5 \mathrm{~m}$ and $3.0 \mathrm{~m}$ respectively, and the physical installation can be show in Fig. 7(a). After the impulse loading on the tower, it will vibrate freely. The result of sampled data is shown in Fig. 7(b). The upper half shows the vibration of the position $1.5 \mathrm{~m}$ upper from the ground while the lower half is $3.0 \mathrm{~m}$ from the ground. The vibration feature of the steel tube tower is shown in Fig. 8.

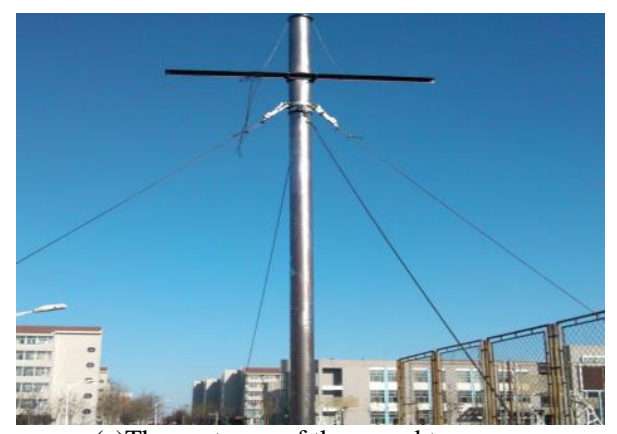

(a)The spot map of the guyed tower

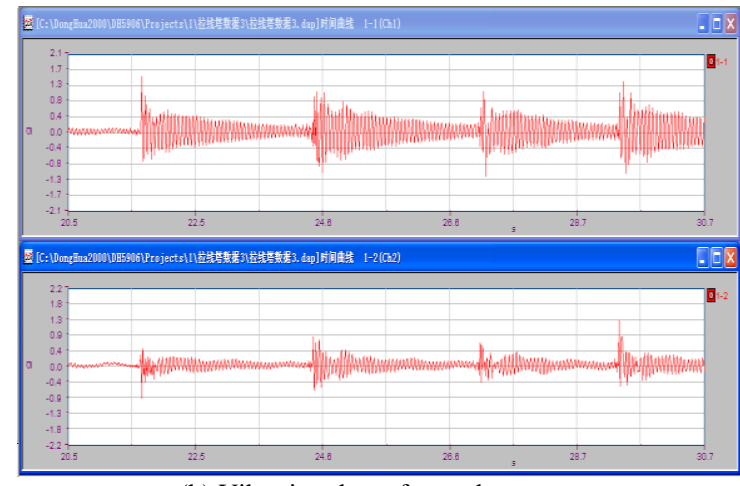

(b) Vibration data of guyed tower

Fig. 5. Experiments of the guyed tower.

The upper half shows the vibration of the position $1.0 \mathrm{~m}$ upper from the ground. And the lower half shows the vibration of the position $4.5 \mathrm{~m}$ upper from the ground.

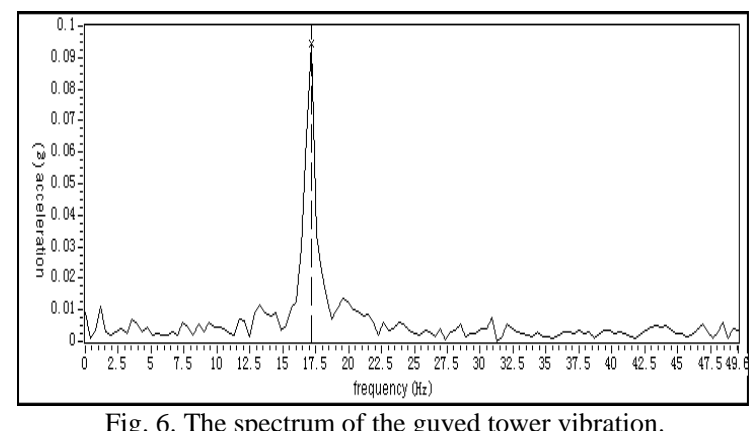

Form the frequency analysis, the analysis of the steel tube tower can gain some apparent vibration features while for the guyed tower, only the first order frequency can be calculated from the analysis. The vibration characteristic of the tower various with the constraint of the tower. In the experiment, meely constaint of ground was applied to the steel tube tower, and the other end of the tower is free. Multi-order frequency can be obtained by frequency domain analysis of the acceleration. While to the guyed tower, whose both ends are constrainted, one order frequency value can be obtained.

The frequencies of the towers reflect some potential problems of the operation status. That means the towers are healthy when the detected natural frequencies of the towers are close to the normal value. Oppositely, changings of the natural frequency illustrate the changing of the system mass distribution, system structure and elements connection, 
which usually means the occurrence of failure. When faults occur, it is necessary to check the towers and repair the malfunction.

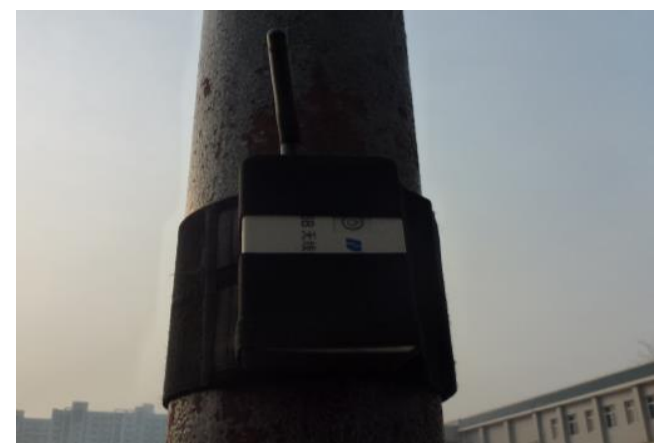

(a) The spot map of the steel tube tower

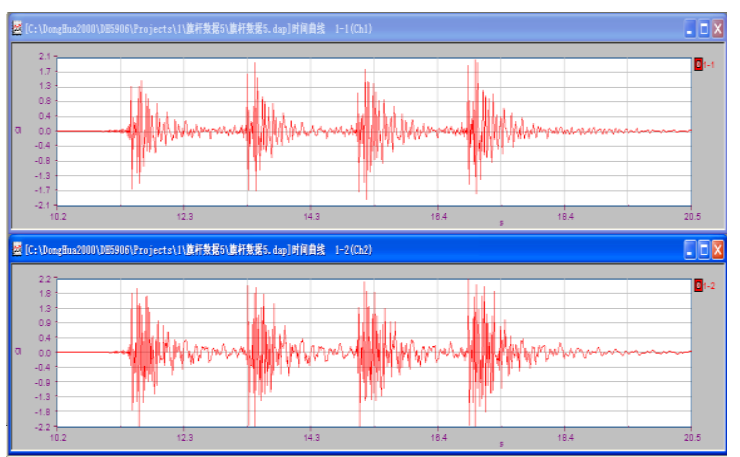

(b) Vibration data of steel tube tower

Fig. 7. Experiments of the steel tube tower.

The upper half shows the vibration of the position $1.5 \mathrm{~m}$ upper from the ground. And the lower half shows the vibration of the position $3.0 \mathrm{~m}$ upper from the ground.

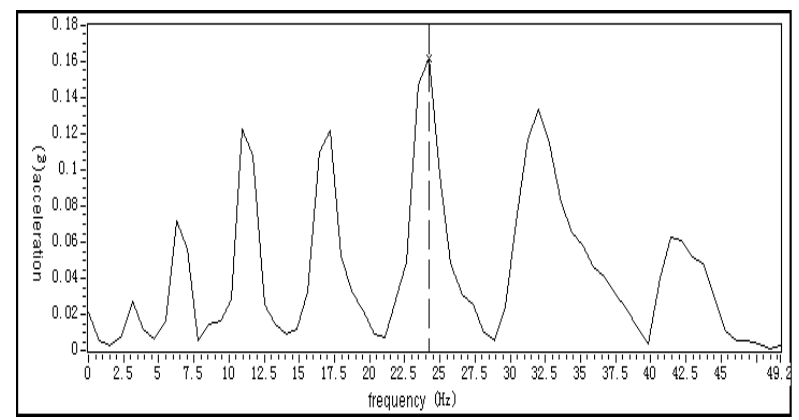

Fig. 8. The spectrum of the steel tube tower vibration.

\section{CONCLUSION}

In this paper, the transmission tower vibration monitoring technology based on the Internet of Things (IoT) is analyzed and studied. A set of vibration monitoring system for transmission tower is designed and implemented in detail. The system mainly contains three segments as network sensing, data communication and system software. The main procedure is compiled on LabVIEW platform and it is able to acquire and analyze the data, remote display etc. To verify the developed system, experiments on guyed tower, are implemented by creating the free vibration after impulse load. Through frequency domain analysis, the first order natural frequency of the guyed tower model can be obtained, and we can judge the health level of the tested model by comparing the real-time monitoring vibration value with the normal value.

\section{REFERENCES}

[1] Q. B. Su, J. Liu, S. L, C. X. Fan, and J. J. Sun, "Literature review of the Internet of Things: Conception, architecture and key technology," Journal of Beijing University of Posts and Telecommunications, vol. 3, pp. 1-9, July 2010.

[2] N. Li, X. Chen, F. Wu, and X. Z. Li, "Information aggregation technology for smart grid based on the internet of things," Information and communication technology, pp. 21-28, 2010.

[3] C. X. Guo, Z. X. Gao, J. J. Zhang, and J. Q. Bi, "Power transmission and transformation equipment condition monitoring and maintenance asset management based on the Internet of Things," Journal of electric power science and technology, vol. 25, pp. 36-41, April 2010.

[4] T. Yin, H. F. Lam, H. M. Chow, and H. P. Zhu, "Dynamic reduction-based structural damage detection of transmission tower utilizing ambient vibration data," Engineering Structures, vol. 31, pp. 2009-2019, September 2009.

[5] H.-F. Lam and T. Yin, "Dynamic reduction-based structural damage detection of transmission towers: Practical issues and experimental verification," Engineering Structures, vol. 33, pp. 1459-1478, May 2011.

[6] S. W. Doebling, C. R. Farrar, and M. B. Prime, "A summary review of vibration-based damage identification methods," Shock and Vibration Digest, vol. 30, pp. 91-105, February 1998.

[7] Z. H. Ning, "The key technology and application study of the internet of things," Computer Programming Skills \& Maintenance, vol. 20, no. 126, pp. 83-84, 2011.

[8] L. Chunli, "Intelligent transportation based on the internet of things," in Proc. 2nd IEEE International Conference on Consumer Electronics, Communications and Networks (CECNet), 2012, pp. 360-362.

[9] X. Z. Li and J. M. Liu, "The technology and application of the internet of things for the Smart Grid," Telecommunications Network Technology, vol. 8, pp. 41-45, 2010.

[10] Z. H. Hou, "The universal design of application layer architecture of the internet of things," The Technology of the Internet of Things, vol. 1, no. 7, pp. 34-36, 2011.

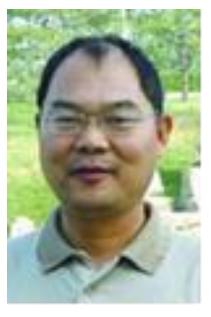

Wang Zhangqi was born in Shanxi Province 1964. He received his Ph.D degree in 1997 from North China Electric Power University. Now he is a professor in Department of Mechanical Engineering in NCEPU. His main research interests include nonlinear dynamics of transmission towers.

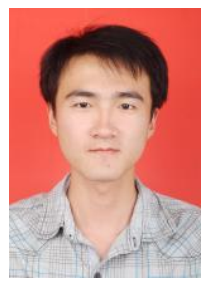

Wang Jian received his B.Sc. degree in 2011 from North China Electric Power University. He received his M.Sc. degree in 2013 from North China Electric Power University. Now he is a doctoral candidate in the Department of Mechanical Engineering in North China Electric Power University. His main research interests include dynamics of transmission towers and its condition monitoring. 